\title{
ABCC10/MRP7 is associated with vinorelbine resistance in non-small cell lung cancer
}

\author{
YUJI BESSHO, TETSUYA OGURI, HIROAKI OZASA, TAKEHIRO UEMURA, HIDEO SAKAMOTO, \\ MIKINORI MIYAZAKI, KEN MAENO, SHIGEKI SATO and RYUZO UEDA
}

\begin{abstract}
Department of Medical Oncology and Immunology, Nagoya City University Graduate School of Medical Sciences, 1 Kawasumi, Mizuho-cho, Mizuho-ku, Nagoya 467-8601, Japan
\end{abstract}

Received August 25, 2008; Accepted October 7, 2008

DOI: $10.3892 /$ or_00000217

\begin{abstract}
The non-small cell lung cancer (NSCLC) cells SK-LC6 and NCI-H23 were continuously exposed to vinorelbine (VNB), and the VNB-resistant clones, SK-LC6/VNB and $\mathrm{H} 23 / \mathrm{VNB}$ were selected. Since SK-LS6/VNB and H23/VNB cells showed cross-resistance to certain anticancer drugs, such as paclitaxel and docetaxel, we examined the gene expression levels of drug efflux transporters of the ATP-binding cassette $(\mathrm{ABC})$ family. We found that the gene expression of $\mathrm{ABCB} 1 / \mathrm{MDR} 1$ and $\mathrm{ABCC} 10 / \mathrm{MRP} 7$ in SKLC6/VNB and H23/VNB cells was increased compared with that in SK-LS6 and NCI-H23 cells, whereas the expression of $\mathrm{ABCC} 1 / \mathrm{MRP} 1, \mathrm{ABCC} 2 / \mathrm{MRP} 2, \mathrm{ABCC} 3 / \mathrm{MRP} 3$ and ABCG2/BCRP did not change among these cells. Treatment with ABCB1/MDR1 inhibitor verapamil and ABCC10/MRP7 inhibitor sulfin-pyrazone altered the sensitivity of SKLC6/VNB cells to vinorelbine. To confirm the ABCC10/ MRP7 activity, we transfected small interfering RNA against ABCC10/MRP7 to ABCC10/MRP7-expressing RERF-LC-AI cells resulting in the decrease of ABCC10/MRP7 expression concomitant with the alteration of VNB cytotoxicity. Moreover, we detected the expression of ABCC10/MRP7 in 12 of 17 NSCLC cells, whereas ABCB1/MDR1 was detected in only 3 of 17 NSCLC cells. These results indicate that ABCC10/MRP7 may confer VNB resistance in NSCLC.
\end{abstract}

\section{Introduction}

Vinorelbine (VNB) is one of new semi-synthesized vinka alkaloids developed in France, of which anti-tumor activity is

Correspondence to: Dr Tetsuya Oguri, Department of Medical Oncology and Immunology, Nagoya City University Graduate School of Medical Sciences, 1 Kawasumi, Mizuho-cho, Mizuho-ku, Nagoya 467-8601, Japan

E-mail: t-oguri@med.nagoya-cu.ac.jp

Key words: non-small cell lung cancer, vinorelbine, ABCB1/ MDR1, ABCC10/MRP7, resistance susceptible mainly to non-small cell lung cancer (NSCLC) and breast cancer. The chemical structure of VNB is characterized by changes in the catharanthine moiety of VNB. Clinically, VNB has mainly been found to be effective in the treatment of advanced non-small cell lung cancer and metastatic breast cancer (1-3). It is assumed that VNB selectively acts on tubulin which elaborates microtubules, strands the cells at G1 phase and interferes with mitosis.

Several mechanisms of resistance to VNB have been reported. CYP3A4 is mainly responsible for the metabolism of VNB (4). High levels of expression of class III B-tubulin in tumor cells is associated with resistance to $\mathrm{VNB}$ and a poor prognosis in patients with NSCLC receiving VNB-based chemotherapy (5). Furthermore, it has also been suggested that reduced drug accumulation mediated by the ATPdependent efflux pump or RLIP76, a non-ABC transporter, mediates VNB transport and is capable of coferring drug accumulation defect and resistance to lung cancer cells (6-10).

Lung cancer is one of the most common malignancies worldwide, and several randomized clinical trials and metaanalyses have demonstrated that chemotherapy can slightly but significantly prolong survival in patients with lung cancer $(11,12)$. VNB is one of the promising agents against human lung cancers $(1,3)$, and resistance to VNB is a common problem in the survival of lung cancer patients. However, biomarkers for predicting resistance to VNB have not been fully identified. To elucidate the biomarkers of resistance to $\mathrm{VNB}$, we developed an in vitro model of resistance to VNB by continuous and progressive exposure of the NSCLC cell line SK-LS6 and NCI-H23 to VNB, and selected the VNBresistant clone SK-LC6/VNB and H23/VNB. Herein we describe the determinants of resistance to $\mathrm{VNB}$ as elucidated using our selected VNB-resistant cells.

\section{Materials and methods}

Cell lines and chemicals. The following human lung cancer cell lines were used in this study: 14 adenocarcinomas (A549, VMRC-LCD, VMRC-LCF, NCI-H23, ACC-LC-174, ACCLC-176, SK-LC-10, RERF-LC-MS, RERF-LC-MT, RERFLC-OK, PC-9, ACC-LC-94 and RERF-LC-AI), 2 squamous cell carcinomas (PC-10 and QG56), and 2 large-cell carcinomas (NCI-H460 and SK-LC6). Cells were cultured in RPMI-1640 (or Dulbecco's modified Eagle's medium) 
Table I. Drug concentrations for $\mathrm{IC}_{50}$ of various cytotoxic drugs in VNB-resistant cells.

\begin{tabular}{|c|c|c|c|c|c|c|}
\hline Drug & $\begin{array}{l}\text { SK-LC6 } \\
\mathrm{IC}_{50}(\mathrm{nM}) 95 \% \mathrm{CI}\end{array}$ & $\begin{array}{l}\text { SK-LC6/VNB } \\
\mathrm{IC}_{50}(\mathrm{nM}) 95 \% \mathrm{CI}\end{array}$ & RR & $\begin{array}{l}\text { NCI-H23 } \\
\mathrm{IC}_{50}(\mathrm{nM}) 95 \% \mathrm{CI}\end{array}$ & $\begin{array}{l}\mathrm{H} 23 / \mathrm{VNB} \\
\mathrm{IC}_{50}(\mathrm{nM}) 95 \% \mathrm{CI}\end{array}$ & RR \\
\hline Vinorelbine & $0.0061(0.0012-0.0091)$ & $182(121-272)$ & 29836 & $0.007(0.0022-0.027)$ & $0.55(0.32-0.92)$ & 78.00 \\
\hline Paclitaxel & $0.065(0.042-0.10)$ & $39.7(25.6-61.8)$ & 610 & $0.036(0.028-0.046)$ & $3.63(2.04-6.48)$ & 100.00 \\
\hline Docetaxel & $0.085(0.0043-0.095)$ & $36.3(23.4-56.1)$ & 576 & $0.099(0.054-0.133)$ & $6.96(1.67-103.6)$ & 70.00 \\
\hline Gemcitabine & $0.56(0.07-1.1)$ & $0.78(0.54-0.11)$ & 1.39 & $0.013(0.009-0.018)$ & $0.010(0.001-0.67)$ & 0.76 \\
\hline Cisplatin & $40050(6903-232400)$ & $42660(5746-316800)$ & 1.06 & $10520(1207-915800)$ & $11660(2395-567700)$ & 1.10 \\
\hline Etoposide & $6235(3599-10800)$ & 4499 (2952-6855) & 0.72 & $1572(1182-2721)$ & $3663(2633-5095)$ & 2.33 \\
\hline SN-38 & $0.24(0.218-0.33)$ & $0.10(0.08-1.31)$ & 0.41 & $0.08(0.069-0.098)$ & $0.53(0.33-0.84)$ & 6.60 \\
\hline
\end{tabular}

$\mathrm{RR}$, resistance ratio: $\left(\mathrm{IC}_{50}\right.$ in resistant subline $) /\left(\mathrm{IC}_{50}\right.$ in the parental cells $)$.

supplemented with $10 \%$ heat-inactivated FBS and $1 \%(\mathrm{v} / \mathrm{w})$ penicillin/streptomycin. SN-38 was a gift from Yakult Honsha, Co., Ltd (Tokyo, Japan). Verapamil and sulfinpyrazone were purchased from Wako Pure Chemical Industries (Osaka, Japan). Gemcitabine was obtained from Eli Lilly Pharmaceuticals (Indianapolis, IN, USA). Cisplatin, etoposide, and paclitaxel were gifts from Bristol Myers (Tokyo, Japan). VNB was obtained from Kyowa Hakko Kogyo (Tokyo, Japan). Docetaxel was obtained from Sanofi Aventis (Tokyo, Japan).

VNB-resistant cell lines were selected by culturing SKLS6 and NCI-H23 cells in the presence of VNB at concentrations of $500 \mathrm{pM}$ and higher for 2 months, with the drugcontaining medium changed every 4-7 days. They were made resistant by continuous exposure to stepwise-increasing concentrations of the drug. Furthermore, variants of SK-LC6 and NCI-H23 cells exhibiting intermediate resistance were cultured by limiting the dilution method for cloning.

Total RNA extraction and RT-PCR. Total RNA was extracted with TRIzol reagent (Invitrogen, USA) according to the manufacturer's instructions. cDNA was synthesized using a random hexamer (Amersham, UK) with Superscript RNase $\mathrm{H}$-reverse transcriptase (Gibco-BRL, USA). The reversetranscribed cDNA from each sample was subjected to PCR amplification using Taq polymerase (Roche Applied Science, France) and primers. The sequences of the ABCC10/MRP7 primers were 5'-gcgggttaagttgtgacaga-3' (forward) and 5'-ccc accogcagaacttga-3' (reverse). The ABCB1/MDR1, ABCC1/ MRP1, ABCC2/MRP2 and ABCC3/MRP3 primers and the PCR conditions have been described previously $(13,14)$. Amplified products were separated by $2 \%$ agarose gel electrophoresis, and bands were visualized by staining with ethidium bromide. We also performed quantitative RT-PCR with the LightCycler FastStart DNA SYBR-Green kit (Roche Applied Science). We conducted a melting-curve analysis to control for the specificity of the amplification products. The number of transcripts was calculated from a standard curve obtained by plotting the known input of six different concentrations against the number of PCR cycles at which the detected fluorescence intensity reached a fixed value. For each sample, results were normalized by the housekeeping gene GAPDH.
Chemosensitivity assay. Cells were cultured at 5,000 cells/ well in 96-well tissue culture plates. To assess cell viability, stepwise 10 -fold dilutions of the anticancer drug were added $2 \mathrm{~h}$ after plating, and the cultures were incubated at $37^{\circ} \mathrm{C}$ for $96 \mathrm{~h}$. At the end of the culture period, $20 \mathrm{ml}$ of MTS [3-(4,5dimethylthiazol-2-yl)-5-(3-carboxymethoxyphenyl)-2-(4sulfophenyl)-2H-tetrazolium, inner salt] solution (CellTiter 96 ${ }^{\circledR}$ AQueous One Solution Cell Proliferation Assay, Promega, Madison, WI, USA) was added and cells were incubated for a further $4 \mathrm{~h}$, after which the absorbance was measured at $490 \mathrm{~nm}$ using an ELISA plate reader. Mean values were calculated from three independent experiments performed in quadruplicate. The chemosensitivity is expressed here as the drug concentration for $50 \%$ cell survival $\left(\mathrm{IC}_{50}\right)$, as determined from the concentration-effect relationship using Graph Pad Prism (version 4, GraphPad Software, USA).

Inhibition of transporter activity by verapamil or sulfinpyrazone. ABCB1/MDR1 and ABCC10/MRP7 expressed SK-LC6/VNB cells $\left(2 \times 10^{5}\right.$ cells $)$ were plated in six-well tissue culture plates. As an ABCB1/MDR1 inhibitor, we used verapamil as referred previously (15). After $24 \mathrm{~h}$, the cells were exposed to the ABCB1/MDR1 inhibitor verapamil for $30 \mathrm{~min}$ and we added VNB in the presence of verapamil. As an ABCC10/MRP7 inhibitor, we used sulfinpyrazone as referred previously (16). After $24 \mathrm{~h}$, the cells were exposed to the ABCB1/MDR1 inhibitor sulfinpyrazone for $30 \mathrm{~min}$ and we added VNB in the presence of sulfinpyrazone.

Statistical modification of vinorelbine cytotoxicity by RNA interference. Approximately $1 \times 10^{5}$ cells were transfected with small interfering RNA(siRNA) oligonucleotide using X-tremeGENE (Roche, Applied Science) to produce a final RNA concentration of $50 \mathrm{nmol} / 1$ in serum-free Opti-MEM medium (Invitrogen) as described previously. At $24 \mathrm{~h}$ after transfection, we changed the medium to RPMI-1640 supplemented with $10 \%$ heat-inactivated FBS. After an additional $24 \mathrm{~h}$, total RNA was extracted, or the cells were treated with VNB for $48 \mathrm{~h}$, and then the viable cells were counted using trypan blue staining to evaluate the cytotoxicity of VNB. The siRNA oligonucleotides for ABCC10/MRP7 (predesigned siRNA, ID 117717) were purchased from Ambion (Austin, 
A

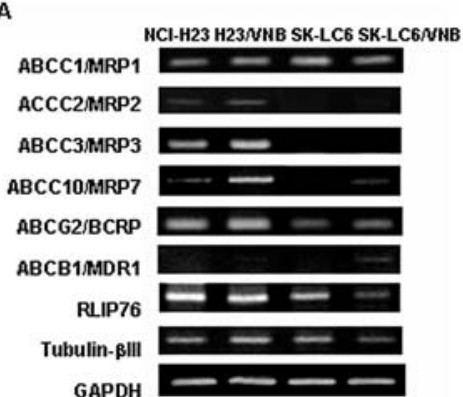

B
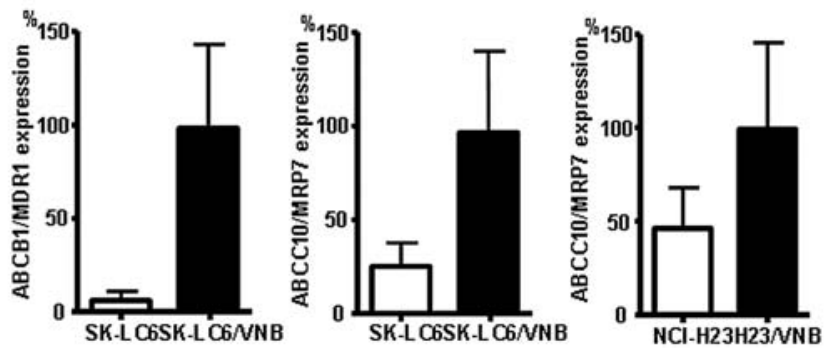

Figure 1. (A) Gene expression levels of the biomarkers of VNB in SKLS6/VNB, SK-LS6, NCI-H23 and H23/VNB cells. (B) Gene expression levels of ABCB1/MDR1 and ABCC10/MRP7 by real-time PCR in SK-LS6/ VNB and SK-LS6 cells and gene expression levels of ABCC10/MRP7 by real-time PCR in $\mathrm{H} 23$ /VNB and $\mathrm{H} 23$ cells.

TX, USA). The negative-control siRNA (Silencer negative control siRNA), which does not exert non-specific effects on gene expression, was also purchased from Ambion.

Intacellular paclitaxel concentration. We treated $6 \times 10^{5}$ of RERF-LC-AI and RERF-LC-AI (ABCC10 siRNA) cells with $20 \mu \mathrm{mol} / 1$ of paclitaxel for $2 \mathrm{~h}$. After thrice washing with cold PBS, the pellet was stored at $-80^{\circ} \mathrm{C}$ until analysis. Paclitaxel concentrations were quantified by a high-performance liquid chromatography (HPLC) assay, as described previously (17).

\section{Results}

Establishment of VNB-resistant cell lines. To obtain VNBresistant variants, we exposed the NSCLC cell line SK-LC6 and NCI-H23 to increasing concentrations of VNB. Exposure of cultures to $500 \mathrm{pM}$ VNB for 4 weeks resulted in the isolation of several VNB-resistant cell lines. One of these sublines was further characterized, and the drug concentration was gradually increased every 2 to 4 weeks. Finally, cells (namely SK-LS6/VNB or H23/VNB) growing vigorously in medium containing $10 \mathrm{nM}$ VNB were obtained 2 months later. The sensitivities of parental and VNB-resistant cells to a panel of chemotherapeutic drugs are summarized in Table I. The $\mathrm{IC}_{50}$ for VNB was 29836 times higher for SK-LC6/ VNB cells $(182 \mathrm{nM})$ than for the parent SK-LC6 cells $(0.0061 \mathrm{nM})$ and $\mathrm{H} 23 / \mathrm{VNB}$ was 78 times higher resistant than the parent NCI-H23 cells. VNB-resistant cells showed cross-resistance to paclitaxel and docetaxel, but not to the other four drugs tested: gemcitabine, etoposide, SN-38, and cisplatin. in $S K-L C 6 / V N B$ and H23/VNB cells. Since SK-LC6/VNB
A

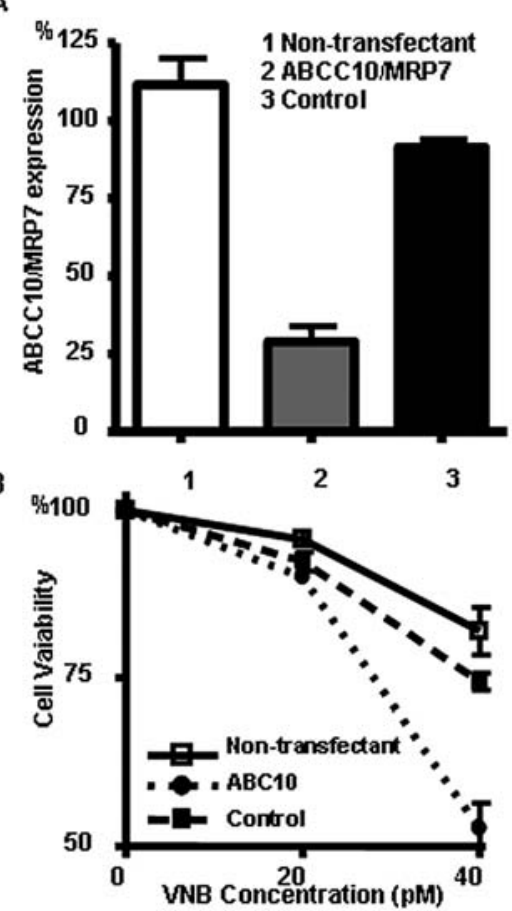

C

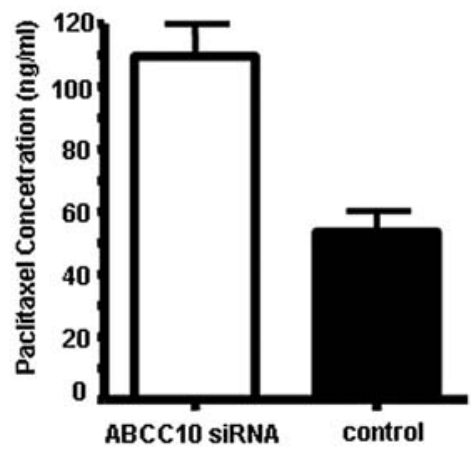

Figure 2. (A) Modification of VNB cytotoxicity by ABCC10/ MRP7 siRNA. Gene expression levels of ABCC10/MRP7 in RERF-LC-AI cells transfected with siRNAs directed against ABCC10/MRP7, and in control siRNA-transfected or non-transfected RERF-LC-AI cells. (B) Cytotoxicity to 20 and $40 \mathrm{pmol} / 1 \mathrm{VNB}$ was measured in RERF-LC-AI cells transfected with siRNAs directed against $\mathrm{ABCC} 10 / \mathrm{MRP} 7$, and in control or nontransfected RERF-LC-AI cells. The viable cells were counted using trypan blue staining to evaluate the cytotoxicity of VNB. The cell viability following VNB treatment for $48 \mathrm{~h}$ differed between RERF-LC-AI cells transfected with ABCC10/MRP7 siRNA and control siRNA transfected or non-transfected RERF-LC-AI cells. (C) The intracellar accumulation of paclitaxel was analyzed between RERF-LC-AI cells transfected with ABCC10/MRP7 siRNA and control siRNA transfected RERF-LC-AI cells with $20 \mu \mathrm{mol} / \mathrm{l}$ of paclitaxel for $2 \mathrm{~h}$.

and $\mathrm{H} 23 / \mathrm{VNB}$ cells showed cross-resistance to taxanes (paclitaxel and docetaxel), we examined the gene expression levels of drug efflux transporters of the ATP-binding cassette (ABC) family. We found that gene expression of ABCB1/ MDR1 and ABCC10/MRP7 in SK-LC6/VNB and H23/VNB cells were increased compared with that in SK-LC6 and NCI$\mathrm{H} 23$ cells, whereas the expression of ABCC1/MRP1, ABCC2/MRP2, ABCC3/MRP3 and ABCG2/BCRP did not change among these cells (Fig. 1A). We confirmed the differences in $\mathrm{ABCB} 1 / \mathrm{MDR} 1$ and $\mathrm{ABCC} 10 / \mathrm{MRP} 7$ gene expression in SK-LC6/VNB and H23/VNB cells compared 
Table II. Inhibition of transporter activity by verapamil or sulfinpyrazone in VNB-resistant cells.

\begin{tabular}{llll}
\hline Drug & \multicolumn{1}{c}{ SK-LC6 } & SK-LC6/VNB \\
$\mathrm{IC}_{50}(\mathrm{nM}) 95 \%$ CI & $182(121-272)$ & 29836 \\
\hline Vinorelbine & $0.0061(0.0012-0.0091)$ & $0.2(0.075-0.551)$ & 85 \\
Vinorelbine + Verapamil & $0.0024(0.0019-0.0179)$ & $0.2(0.69-1.19)$ & 1166 \\
Vinorelbine + Sulfinpyrazone & $0.0078(0.0009-0.179)$ & $\mathrm{IC}_{50}(\mathrm{nM}) 95 \% \mathrm{CI}$ & \\
\hline
\end{tabular}

$\mathrm{RR}$, resistance ratio: ( $\mathrm{IC}_{50}$ in resistant subline $) /\left(\mathrm{IC}_{50}\right.$ in the parental cells).



Figure 3. Gene expression levels of ABCB1/MDR1 and ABCC10/MRP7 in 17 NSCLC cell lines by RT-PCR: (1) A549, (2) VMRC-LCD, (3) VMRC-LCF, (4) NCI-H23, (5) ACC-LC-174, (6) ACC-LC-176, (7) SK-LC-10, (8) RERF-LC-MS, (9) RERF-LC-MT, (10) RERF-LC-OK, (11) PC-9, (12) ACC-LC-94, (13) NCI-H460, (14) SK-LC6, (15) PC10, (16) QG56 and (17) RERF-LC-AI.

with parental cells by real-time PCR. ABCB1/MDR1 and ABCC10/MRP7 gene expression were increased in SK-LC6/ VNB cell (Fig. 1B). ABCC10/MRP7 gene expression was increased in H23/VNB cell (Fig. 1B). ABCB1/MDR1 gene expression was not detected in $\mathrm{H} 23 / \mathrm{VNB}$ cell. Further, we could not detect an expression of CYP3A4 among these cells (data not shown), the differences in expression of class III ß-tubulin and RLIP76 among these cells (Fig. 1A).

Inhibition of $V N B$ cytotoxicity by verapamil or sulfinpyrazone. To elucidate whether ABCB1/MDR1 actually affects the sensitivity to VNB, we pretreated SK-LC6/VNB cells with verapamil and then measured the VNB cytotoxicity. We found that the sensitivity of SK-LC6/VNB cells to VNB was increased by $30-$ min pretreatment with $20 \mu \mathrm{M}$ verapamil (resistance ratio for VNB decreased from 29836 to 85), whereas that of the parental SK-LC6 cells did not change (Table II). To elucidate whether ABCC10/ MRP7 actually affects the sensitivity to VNB, we pretreated SK-LC6/VNB cells with sulfinpyrazone and then measured the VNB cytotoxicity. We found that the sensitivity of SK-LC6/VNB cells to VNB was increased by 30 -min pretreatment with $30 \mu \mathrm{M}$ sulfinpyrazone (resistance ratio for VNB decreased from 29836 to 1166), whereas that of the parental SK-LC6 cells did not change (Table II).

Statistical modification of VNB cytotoxicity in RERF-LC-AI by ABCC10 siRNA. To investigate functional activity of ABCC10, we used RERF-LC-AI cell which expressed ABCC10/MDR1, but did not express ABCB1/MDR1 (as shown in Fig. 3, lane 17). RERF-LC-AI cells were transfected with siRNAs directed against $\mathrm{ABCC} 10$, and control siRNA-transfected or untransfected cells were used as controls. Cells were harvested at $48 \mathrm{~h}$ after transfection, and extracts we prepared and analyzed by quantitative realtime RT-PCR. The expression levels of ABCC10 gene were decreased at $48 \mathrm{~h}$ after siRNA transfection (Fig. 2A). We then investigated whether ABCC10 siRNA would enhance the cytotoxicity to VNB in RERF-LC-AI cells. Following 48-h transfection with ABCC10 siRNA or negative control siRNA or with no transfection, RERF-LC-AI cells were treated with 20 or $40 \mathrm{pmol} / \mathrm{l} \mathrm{VNB}$ for $48 \mathrm{~h}$. The cytotoxicity to VNB was significantly higher for cells exposed to VNB in the presence of ABCC10 siRNA than for cells transfected with control siRNA and for untransfected cells (Fig. 2B). Since negative control siRNA does not affect gene expression, these results indicate that the decrease of expression of ABCC10/MRP7 by siRNA altered the cytotoxicity to VNB.

Intacellular paclitaxel concentration in RERF-LC-AI. Paclitaxel was used to detect the functional activity of ABCC10/MRP7 as a transporter, and paclitaxel is a good substrate for ABCC10/MRP7. We treated 6x105 RERF-LCAI and RERF-LC-AI (ABCC10/MRP7 siRNA) cells with $20 \mu \mathrm{mol} / \mathrm{l}$ of paclitaxel for $2 \mathrm{~h}$. After thrice washing with cold PBS, paclitaxel concentration was detected. The intracellar accumulation of paclitaxel was markedly higher in RERF-LC-AI (ABCC10 siRNA) cells than in RERF-LC-AI cells (Fig. 2C).

Expression of ABCB1 and ABCC10 genes in NSCLC cells. Using RT-PCR, we detected the expression of ABCC10 in 12 of 17 NSCLC cells, whereas ABCB1 was detected in only 3 of 17 NSCLC cells (Fig. 3).

\section{Discussion}

In this study, we successfully isolated VNB-resistant clones by continuously exposing SK-LC6 cells and NCI-H23 cells to VNB. Anticancer drug resistance is often attributed to $\mathrm{ABC}$ transporters in cancer cells, and the two VNB-resistant cell types showed enhanced ABCC10/MRP7 expression. $\mathrm{ABCC} 10 / \mathrm{MRP} 7$ is also overexpressed in vincristine-resistant and paclitaxel-resistant cells $(17,18)$. Taken together with our 
findings, similarly to ABCB1/MDR1, ABCC10/MRP7 may act as an inducible transporter confering resistance to anticancer argents.

Multidrug resistance is often attributed to ABC transporters in cancer cells. ABCB1/MDR1 was the first of the $\mathrm{ABC}$ transporters to be identified and characterized, and the isolation of the second distantly related $\mathrm{ABCC} 1$ transporter facilitated the discovery of eight more genes. It was shown that ABCC10/MRP7 is capable of conferring high levels of resistance (9-13-fold) to docetaxel, a microtubule stabilizing agent. In addition, 3-4-fold levels of resistance were observed for another taxane, paclitaxel, as well as for the microtubule destabilizing agents, vincristine and vinblastine (10). ABCC10/MRP7 is at early stages of investigation. The topology of $\mathrm{ABCC} 10 / \mathrm{MRP} 7$ is similar to that of $\mathrm{ABCC} 1 /$ MRP1, ABCC2/MRP2, ABCC3/MRP3, and ABCC6/MRP6, in that it possesses three membrane spanning domains and two nucleotide-binding domains. Studies on the expression of $\mathrm{ABCC} 10 / \mathrm{MRP} 7$ protein in tissues have not yet been reported. Whether ABCC10/ MRP7 contributes significantly to the in vivo sensitivity of normal tissues or tumor is unknown, but it is reasonable to speculate that it may play a role in the intrinsic sensitivity of tissues and tumors in which it is expressed. ABCC10/MRP7 transcript was detected in the HepG2 liver cancer cell line and in two prostate cancer cell lines, CWR22Rv1 and TSU-PR1 (19). In addition, the transcript was expressed in eight tumor specimens, including breast, lung, colon, prostate, ovary, and pancreas (20).

It has been reported that the expression of $\mathrm{ABCB} 1 /$ MDR1 gene product, $\mathrm{P}$-glycoprotein is correlated with multidrug resistance associated with $\operatorname{VNB}(7,8,21)$. In the present study, we found that the ABCB1/MDR1 inhibitor verapamil or $\mathrm{ABCC} 10 / \mathrm{MRP} 7$ inhibitor sulfinpyrazonemediated sensitivity to VNB in SK-LC6/VNB cells, which express ABCB1/MDR1 and ABCC10/MRP7. We considered that $\mathrm{ABCB} 1 / \mathrm{MDR} 1$ or $\mathrm{ABCC} 10 / \mathrm{MRP} 7$ may have a possibility of becoming the key molecule of VNB-based chemotherapy. However, we did not examine the affinity of ABCB1/MDR1 or ABCC10/MRP7 to VNB, so it was not elucidated whether ABCB1/MDR1 or ABCC10/MRP7 is more significant in aquired resistance to $\mathrm{VNB}$. The efflux pump function of $\mathrm{ABCB} 1 / \mathrm{MDR} 1$ or $\mathrm{ABCC} 10 / \mathrm{MRP} 7$ is different. $\mathrm{ABCB} 1 / \mathrm{MDR} 1$ gene product $\mathrm{P}$-glycoprotein is a membrane protein that functions as an ATP-dependent efflux pump, transporting exogenous and endogenous substrates from inside to the outside of cells. Physiological expression of $\mathrm{P}$-glycoprotein in tissues with excretory or protective function is a major determinant of drug disposition and provides a cellular defense mechanism against potentially harmful compounds, whereas ABCC10/MRP7 is a lipophilic anion pump that is able to confer resistance to chemotherapeutic agents.

Using RT-PCR, we detected the expression of ABCC10/ MRP7 in 12 of 17 NSCLC cells, whereas ABCB1/MDR1 was detected in only 3 of 17 NSCLC cells. Lai et al (22) measured expression of ABCB1/MDR1 in lung cancers of all major histological types as well as corresponding normal lung tissues and tumor cell lines. In most of these tumors, the expression ABCB1/MDR1 mRNA was low or undetectable. We considered that ABCC10/MRP7 has more effect to inherent resistance to VNB-based chemotherapy compared with $\mathrm{ABCB} 1 / \mathrm{MDR} 1$. These results indicate the importance of ABCC10/MRP7 for VNB efflux in NSCLC cells. We elucidated that a decreased expression of ABCC10/ MRP7 by siRNA altered the cytotoxicity to VNB in RERF-LC-AI cells, which express ABCC10/MRP7, and less ABCB1/ MDR1.

For the detection of functional activity of ABCC10/ MRP7 as a transporter, the intracellar accumulation of paclitaxel was observed instead of VNB. The intracellar accumulation of paclitaxel was markedly higher when ABCC10/MRP7 expression was decreased by siRNA in RERF-LC-AI cells. It is reasonable to consider that $\mathrm{ABCC} 10 / \mathrm{MRP} 7$ as a transporter, reduced accumulation of VNB in NSCLC. These results indicate that the expression levels of ABCC10/MRP7 may be associated with VNB activity in NSCLC.

In conclusion, the present study shows that the expression level of ABCC10/MRP7 may be a useful determinant for VNB activity in NSCLC. Future studies should investigate the clinical and functional involvement of ABCC10/MRP7 in resistance to $\mathrm{VNB}$ in vivo.

\section{References}

1. Zhou XJ and Rahmani R: Preclinical and clinical pharmacology of vinca alkaloids. Drugs 44: 1-16, 1992.

2. Goa KL and Faulds D: Vinorelbine. A review of its pharmacological properties and clinical use in cancer chemotherapy. Drugs Aging 5: 200-234, 1994.

3. Toso $\mathrm{C}$ and Lindley C: Vinorelbine: a novel vinca alkaloid. Am J Health Syst Pharm 52: 1287-1304, 1995.

4. Kajita J, Kuwabara T, Kobayashi H and Kobayashi S: CYP3A4 is mainly responsibile for the metabolism of a new vinca alkaloid, vinorelbine, in human liver microsomes. Drug Metab Dispos 28: $1121-1127,2000$

5. Sève P, Mackey J, Isaac S, et al: Class III beta-tubulin expression in tumor cells predicts response and outcome in patients with non-small cell lung cancer receiving paclitaxel. Mol Cancer Ther 4: 2001-2007, 2005.

6. Stuckler D, Singhal J, Singhal SS, Yadav S, Awasthi YC and Awasthi S: RLIP76 transports vinorelbine and mediates drug resistance in non-small cell lung cancer. Cancer Res 65: 991-998, 2005.

7. Dumontet C and Sikic BI: Mechanisms of action of and resistance to antitubulin agents: microtubule dynamics, drug transport, and cell death. J Clin Oncol 17: 1061-1070, 1999.

8. Adams DJ and Knick VC: P-glycoprotein mediated resistance to 5'-nor-anhydro-vinblastine (Navelbine). Invest New Drugs 13: 13-21, 1995.

9. Kruh GD and Belinsky MG: The MRP family of drug efflux pumps. Oncogene 22: 7537-7552, 2003.

10. Hopper-Borge E, Chen ZS, Shchaveleva I, Belinsky MG and Kruh GD: Analysis of the drug resistance profile of multidrug resistance protein 7 (ABCC10): resistance to docetaxel. Cancer Res 64: 4927-4930, 2004.

11. Non-Small Cell Lung Cancer Collaborative Group: Chemotherapy in non-small cell lung cancer. A meta-analysis using updated data on individual patients from 52 randomized clinical trials. BMJ 311: 899-909, 1995.

12. Schiller JH, Harrington D, Belani CP, et al: Comparison of four chemotherapy regimens for advanced non-small-cell lung cancer. N Engl J Med 346: 92-98, 2002.

13. Hirata S, Katoh O, Oguri T, Watanabe H and Yajin K: Expression of drug resistance-related genes in head and neck squamous cell carcinomas and normal mucosa. Jpn J Cancer Res 91: 84-90, 2000.

14. Oguri T, Isobe T, Fujitaka K, Ishikawa N and Kohno N: Association between expression of the MRP 3 gene and exposure to platinum drugs in lung cancer. Int J Cancer 93: 584-589, 2001.

15. Etiévant C, Pauwels $\mathrm{O}$ and Kiss R: Digital cell image analysis of verapamil-induced effects in chemosensitive and chemoresistant neoplastic cell lines. J Cancer Res Clin Oncol 120: 76-84, 1993. 
16. Chen ZS, Hopper-Borge E, Belinsky MG, Shchaveleva I, Kotova E and Kruh GD: Characterization of the transport properties of human multidrug resistance protein 7 (MRP7 ABCC10). Mol Pharmacol 63: 351-358, 2003.

17. Oguri T, Ozasa H, Uemura T, et al: MRP7/ABCC10 expression is a predictive biomarker for the resistance to paclitaxel in nonsmall-cell lung cancer. Mol Cancer Ther 7: 1150-1155, 2008.

18. Naramoto $H$, Uematsu $T$, Uchihashi $T$, et al: Multidrug resistance-associated protein 7 expression is involved in crossresistance to docetaxel in salivary gland adenocarcinoma cell lines. Int J Oncol 30: 393-401, 2007.

19. Dabrowska M and Sirotnak FM: Regulation of transcription of the human MRP7 gene. Characteristics of the basal promoter and identification of tumor-derived transcripts encoding additional 5' end heterogeneity. Gene 341: 129-139, 2004.
20. Takayanagi S, Kataoka T, Ohara O, Oishi M, Kuo MT and Ishikawa T: Human ATP-binding cassette transporter ABCC10: expression profile and p53-dependent upregulation. J Exp Ther Oncol 4: 239-246, 2004.

21. Stavrovskaya AA: Cellular mechanisms of multidrug resistance of tumor cells. Biochemistry 65: 95-106, 2000

22. Lai SL, Goldstein LJ, Gottesman MM, et al: MDR1 gene expression in lung cancer. J Natl Cancer Inst 81: 1144-1150, 1989. 